\title{
Association of cortactin, fascin-1 and epidermal growth factor receptor (EGFR) expression in ovarian carcinomas: Correlation with clinicopathological parameters
}

\author{
Chih-kung Lin ${ }^{\mathrm{a}}$, Her-Young Su ${ }^{\mathrm{b}}$, Wen-Chiuan Tsai ${ }^{\mathrm{a}}$, Lai-Fa Sheu ${ }^{\mathrm{a}}$ and Jong-Shiaw Jin ${ }^{\mathrm{a}, *}$ \\ ${ }^{a}$ Department of Pathology, Tri-Service General Hospital, National Defense Medical Center, Taipei, Taiwan \\ ${ }^{\mathrm{b}}$ Department of Gynecology \& Obstetrics, Tri-Service General Hospital; National Defense Medical Center, Taipei, \\ Taiwan
}

\begin{abstract}
Cortactin, fascin-1 and EGFR are recognized as important factors in tumor progression. We tested the hypothesis that cortactin, fascin-1 and EGFR expression correlates with clinicopathological parameters of the four most common ovarian surface epithelial carcinomas - serous cystadenocarcinoma, mucinous cystadenocarcinoma, endometrioid adenocarcinoma, and clear cell carcinoma. Immunohistochemical analysis of cortactin, fascin-1 and EGFR was performed using tissue microarrays of 172 specimens comprising 69 serous cystadenocarcinomas, 44 mucinous cystadenocarcinomas, 45 endometrioid adenocarcinomas and 14 clear cell carcinomas. All ovarian carcinomas showed significant expression of cortactin, fascin-1 and EGFR in staining intensity, tumor percentages and immunostaining scores. In addition, higher immunostaining scores of fascin- 1 correlated with more advanced cancer stages (TNM), poorer histological differentiation and poorer survival rate of mucinous cystadenocarcinoma. Similarly, higher immunostaining scores of cortactin correlated with T stages and histological differentiation of serous cystadenocarcinoma. The immunostaining scores of EGFR did not correlate with TNM stages, tumor differentiation or prognosis in the four ovarian surface epithelial carcinomas. Our findings suggest that cortactin and fascin-1 may serve as good biomarkers in evaluating aggressiveness of ovarian serous and mucinous cystadenocarcinoma. And the pharmacological inhibitors of fascin-1 activity may slow down tumor progression and prolong survival time in patients with mucinous cystadenocarcinoma.
\end{abstract}

Keywords: Cortactin, fascin-1, EGFR, immunostaining score, ovarian carcinomas

\section{Introduction}

Cancer of the ovary represents about $30 \%$ of all cancers of the female genital organs. In developed countries ovarian cancer is about as common as cancers of the corpus uteri $(35 \%)$ and invasive cancer of the cervix $(27 \%)$. The age-adjusted incidence rates vary

\footnotetext{
*Corresponding author: Jong-Shiaw Jin, M.D., Ph.D., Department of Pathology, Tri-Service General Hospital, National Defense Medical Center, No. 325, Sec. 2, Cheng-Gong Road, Taipei, Taiwan Tel.: +886 2 87923311, Ext. 16731; Fax: +886 2 87927159; E-mail: jsjin@ndmctsgh.edu.tw.
}

from fewer than two new cases per 100,000 women in most of Southeast Asia and Africa to over 15 cases per 100,000 in Northern and Eastern Europe. Migration of tumor cells outside the ovarian capsule accounts for a significant percentage of treatment failures in patients with ovarian malignancies $[7,18,27]$ and degradation of basement membrane by matrix metalloproteinases (MMPs) is one of the most critical steps in various stages of tumor disease progression, including tumor angiogenesis, tumor growth, as well as local invasion and subsequent distant metastasis. In metastatic ovarian carcinoma, cancer cells produced more MMP-2, and MMP-9 and had the poorest prognosis [10]. In 
one study, the effect of fascin on cell invasion also depended on activation of MMP-2 and MMP-9 [42]. Although the detailed pathway is not established, we hypothesized that some relationships existed between cortactin, fascin-1 and EGFR expression and ovarian carcinomas.

Fascin- 1 and cortactin are two important components among actin cross-linking proteins [14,22]. Fascin, an actin-binding protein, is involved in the rearrangement of the cytoskeleton and promotes cellular motility [28]. Fascin-actin interactions are regulated by extracellular matrix, peptide factors, and other actin-binding proteins [1]. In the human body, the genome encodes three subtypes of the fascin family, including fascin-1, fascin2 and fascin-3 [14]. Expression of fascin-1 in normal epithelia is either absent or low [21,43]. And fascin-1 is markedly up-regulated in several different types of tumors, including breast [13], skin [11], lung [25,26], colon [19], brain [28], ovary [17], urinary bladder [34] and gastric malignancies [33].

Cortactin is an actin-binding protein that activates the Arp $2 / 3$ complex to regulate the actin cytoskeleton $[9,36]$ and inhibit de-branching of dendritic actin networks [39]. The gene responsible for cortactin expression is in the chromosome $11 \mathrm{q} 13$ region and is frequently amplified in some human cancers, such as breast, head/neck carcinomas and gastric adenocarcinoma $[23,31,33]$.

The epidermal growth factor receptor (EGFR; ErbB1 ; HER 1 in humans) is a tyrosine kinase receptor, a cellsurface receptor for members of the epidermal growth factor family (EGF-family) of extracellular protein ligands. The EGFR is a member of the ErbB family of receptors, a subfamily of four closely related receptor tyrosine kinases: EGFR (ErbB-1), HER2/c-neu (ErbB2), Her 3 (ErbB-3) and Her 4 (ErbB-4). EGFR exists on the cell surface and is activated by binding of its specific ligands, including epidermal growth factor and transforming growth factor $\alpha(\mathrm{TGF} \alpha)$. EGF receptor activation induces a variety of changes in intracellular physiology, including activation of $\mathrm{Na}^{+} / \mathrm{H}^{+}$transporter [30], oncogene expression [12], stimulation of DNA synthesis and cell proliferation [8], among other changes.

Mutations involving EGFR could lead to its constant activation which could result in uncontrolled cell division - a predisposition for cancer. Consequently, mutations of EGFR have been identified in several types of cancer, and the identification of EGFR as an oncogene has led to the development of anticancer therapeutics directed against EGFR, including gefitinib and erlotinib for lung cancer, and cetuximab for colon cancer. Previous studies have suggested that high levels of EGFR expression are a marker for bad prognosis in ovarian cancer patients [4-6]. In contrast, a published study by Henzen-Logmans et al. showed that EGFR over-expression only occurs in about $12 \%$ of ovarian carcinomas [16]. Schilder and Andrew et al. detected mutations in the TK domain region in 2 of $56(3.6 \%)$ of ovarian adenocarcinomas and observed that a patient on the clinical trial with a mutation in the catalytic domain of EGFR responded to gefitinib, suggesting a method to pre-select a subset of patients whose tumors may be more responsive to this EGF receptor-targeted therapy. While this mutation is a relatively rare event, but this finding could have a dramatic impact on clinical care, and have a profound effect for some ovarian cancer patients. However, the relationship between cortactin, fascin-1 and EGFR expression and clinicopathological parameters of the four most common ovarian carcinomas remains vague.

In this study, we tested the hypothesis that higher expression of cortactin, fascin-1 and EGFR in those patients with the most common ovarian carcinomas correlate with clinicopathological parameters and are associated with advanced cancer stages. We set out to test the hypothesis that increased cortactin, fascin-1 and EGFR immunostaining scores correlate with advanced histological grades, advanced clinical stages and a poorer survival rate for ovarian carcinoma patients

\section{Materials and methods}

Paraffin-embedded tumor tissues were obtained and tissue microarray slides were constructed. Tissue microarrays consisted of samples from 172 patients with primary ovarian tumors, including 69 serous cystadenocarcinomas, 44 mucinous cystadenocarcinomas, 45 endometrioid adenocarcinomas and 14 clear cell carcinomas.

The histopathological differentiation or clinical stage was determined according to TNM (WHO criteria) and FIGO staging systems. Stage T1 was defined as a tumor limited to ovaries. Stage T2 was defined as a tumor involving one or both ovaries with pelvic extension. Stage T3 was defined as a tumor involving one or both ovaries with microscopically confirmed peritoneal metastasis outside the pelvis. One core tissue sample was taken from a selected area of each paraffinembedded tumor tissue, and tissue microarray slides were constructed. Each representative core sample in 
the tissue microarray slide was $2 \mathrm{~mm}$ in diameter. The pathological diagnosis in each case was reviewed by two experienced pathologists. None of the patients had received chemotherapy before surgery.

\subsection{Immunohistochemistry}

Tissue microarray sections were dewaxed in xylene, rehydrated in alcohol, and immersed in 3\% hydrogen peroxide for 10 minutes to suppress endogenous peroxidase activity. Antigen retrieval was performed by heating $\left(100^{\circ} \mathrm{C}\right)$ each section for 30 minutes in 0.01 mol/L sodium citrate buffer ( $\mathrm{pH}$ 6.0). After 3 rinses in phosphate buffered saline (PBS) for 5 minutes, each section was incubated for 1 hour at room temperature with a mouse monoclonal anti-human fascin-1 antibody (NeoMarkers, Freemont, CA, USA, 1:100), a polyclonal mouse anti-human cortactin antibody (1:100; Santa Cruz Biotechnology, Santa Cruz, CA) and a mouse monoclonal anti-human EGFR antibody (DAKO, clone E30, 1:25) diluted in PBS. After 3 washes in PBS for 5 minutes, each section was incubated with horseradish peroxidase-labeled rabbit anti-mouse immunoglobulin (DAKO, Carpinteria, CA, USA) for 1 hour at room temperature. After 3 additional washes, peroxidase activity was visualized with a solution of diaminobenzidine (DAB) at room temperature.

To evaluate immunoreactivity and histological appearance, all tissue microarray experiments were repeated 2 times and slides were examined and scored by 2 experienced pathologists. The intensity of cytoplasmic and membrane immunostaining of tumor cells was scored on a scale of 0 (no staining) to 3 (strongest intensity), and the percentage of tumor cells with cytoplasmic or membranous staining at each intensity was estimated. The percentage of cells (from 0 to 100) at each intensity was multiplied by the corresponding immunostaining intensity (from 0 to 3 ) to obtain an immunostaining score ranging from 0 to 300 .

\subsection{Statistical analysis}

All results are expressed as mean \pm standard error of the mean (SEM). Immunostaining scores of cortactin, fascin-1 and EGFR for different types of ovarian carcinomas were compared to the score for normal ovarian epithelia. Statistical analysis was performed using Student's $t$-test and calculated with Pearson Product Method Correlation test to analyze the relationships between expression of these three biomarkers and clinicopathological parameters of the four most common ovar- ian carcinomas. Statistical significance was defined as a $P$ value of less than 0.05 . In addition, survival time of subjects was calculated from the date of surgery to the date of death. Sixty nine of serous cystadenocarcinoma and forty four of mucinous cystadenocarcinoma patients in this study received 5-year follow up; subjects were divided into two groups in order to compare survival times with cortactin and fascin-1 immunostaining scores. Statistical analysis of survival time was done using the Kaplan-Meier survival test.

\section{Results}

Immunohistochemical staining patterns of the different ovarian carcinomas are presented in Table 1 and representative samples are illustrated in Fig. 1.

\subsection{Immunoscores of fascin-1 correlate with histological grades and clinical stages of mucinous cystadenocarcinoma}

Among 172 ovarian tumors, fascin-1 immunostaining scores were significantly higher in the four ovarian epithelial carcinomas ( $73 \pm 10$ for serous cystadenocarcinoma; $26 \pm 7$ for mucinous cystadenocarcinoma; $63 \pm 13$ for endometrioid adenocarcinoma and $35 \pm 12$ for clear cell carcinoma; all P values $<0.05$ ) (Table 1). Staining of fascin-1 was either very low or absent in normal ovarian epithelial cells. Thirty-seven (84\%) of 44 mucinous cystadenocarcinomas were well differentiated, 4 (9\%) were moderately differentiated, and 3 $(6 \%)$ were poorly differentiated. Additional information, including TNM and AJCC clinical staging distribution, is listed in Table 2. Using the Pearson Product Method Correlation test, higher immunostaining scores of fascin-1 showed a positive correlation with histological grading, AJCC clinical stage, T stages and $\mathrm{N}$ stages, but not with $\mathrm{M}$ stages (Table 2; Fig. 2). In addition, no significant relationships were seen between fascin-1 immunostaining scores and clinicopathological parameters in the other three ovarian epithelial carcinomas, which included serous, endometrioid and clear cell carcinoma (data not shown).

\subsection{Immunoscores of cortactin correlate with histological grades and $T$ stages of serous cystadenocarcinoma}

The cortactin immunostaining scores were significantly higher in four ovarian epithelial carcinomas (261 
Table 1

Immunostaining scores for each marker in different ovarian tumors

\begin{tabular}{lccc}
\hline Ovarian tumor & Intensity & Staining $(\%)$ & Total score \\
\hline Fascin-1 expression & & & \\
Serous cystadenoCA $(n=69)$ & $1.5 \pm 0.1^{*}$ & $37 \pm 4^{*}$ & $73 \pm 10^{*}$ \\
Mucinous cystadenoCA $(n=44)$ & $0.6 \pm 0.1^{*}$ & $14 \pm 3^{*}$ & $26 \pm 7^{*}$ \\
Endometrioid adenoCA $(n=45)$ & $1.4 \pm 0.1^{*}$ & $30 \pm 5^{*}$ & $63 \pm 13^{*}$ \\
Clear cell CA $(n=14)$ & $1.5 \pm 0.1^{*}$ & $22 \pm 6^{*}$ & $35 \pm 12^{*}$ \\
normal surface epithelia $(n=10)$ & $0.1 \pm 0.1$ & $1.3 \pm 1$ & $0.6 \pm 0.4$ \\
EGFR expression & & & \\
Serous cystadenoCA $(n=69)$ & $1.5 \pm 0.1^{*}$ & $93 \pm 2^{*}$ & $144 \pm 9^{*}$ \\
Mucinous cystadenoCA $(n=44)$ & $1.3 \pm 0.1^{*}$ & $70 \pm 6^{*}$ & $104 \pm 13^{*}$ \\
Endometrioid adenoCA $(n=45)$ & $1.6 \pm 0.1^{*}$ & $96 \pm 2^{*}$ & $151 \pm 12^{*}$ \\
Clear cell CA $(n=14)$ & $1.4 \pm 0.2^{*}$ & $64 \pm 10^{*}$ & $106 \pm 21^{*}$ \\
normal surface epithelia $(n=10)$ & $0.4 \pm 0.2$ & $3 \pm 2$ & $3 \pm 2$ \\
Cortactin expression & & & \\
Serous cystadenoCA $(n=69)$ & $2.7 \pm 0.1^{*}$ & $97 \pm 1^{*}$ & $261 \pm 7^{*}$ \\
Mucinous cystadenoCA $(n=44)$ & $2.2 \pm 0.1^{*}$ & $79 \pm 5^{*}$ & $196 \pm 16^{*}$ \\
Endometrioid adenoCA $(n=45)$ & $2.7 \pm 0.1^{*}$ & $97 \pm 1^{*}$ & $261 \pm 7^{*}$ \\
Clear cell CA $(n=14)$ & $2.7 \pm 0.1^{*}$ & $100 \pm 0^{*}$ & $267 \pm 11^{*}$ \\
normal surface epithelia $(n=10)$ & $0.3 \pm 0.2$ & $3 \pm 2$ & $2 \pm 2$ \\
\hline Abbrial
\end{tabular}

Abbreviations: serous cystadenocarcinoma, serous cystadeno CA; mucinous cystadenocarcinoma, mucinous cystadeno CA; endometrioid adenocarcinoma, endometrioid adeno CA; clear cell carcinoma, clear cell CA.

Data are the means \pm standard error of the mean (SEM) of immunostaining scores for each marker in ovarian tumors and normal surface epithelia. ${ }^{*} p<$ 0.05 vs. normal surface epithelia.

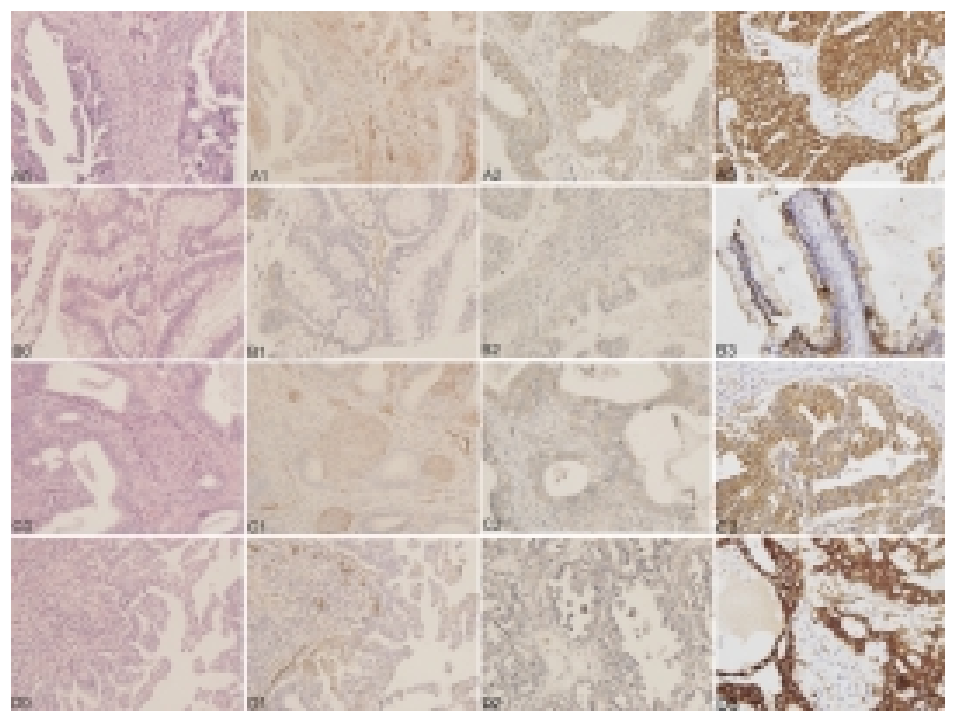

Fig. 1. Hematoxylin and eosin, fascin-1, EGFR and cortactin staining of serous adenocarcinoma (A0, A1, A2, A3), mucinous adenocarcinoma (B0, B1, B2, B3), endometrioid adenocarcinoma (C0, C1, C1, C2, C3), and clear cell carcinoma (D0, D1, D2, D3). Original magnification X 400 .

\pm 7 for serous cystadenocarcinoma; $196 \pm 16$ for mucinous cystadenocarcinoma; $261 \pm 7$ for endometrioid adenocarcinoma and $267 \pm 11$ for clear cell carcino$\mathrm{ma}$; all $\mathrm{P}$ values $<0.05$ ) (Table 1). Among 69 serous cystadenocarcinomas, $41(60 \%)$ were well differentiated, 14 (20\%) were moderately differentiated and 14
(20\%) were poorly differentiated. Additional informations, including TNM and AJCC clinical staging distribution, are listed in Table 3. Using the Pearson Product Method Correlation test, higher immunostaining scores of fascin-1 showed a positive correlation with histological grading and $\mathrm{T}$ stages, but not with AJCC, $\mathrm{N}$ and 
Table 2

Total score of Fascin-1 immunostaining and clinicopathological parameters of mucinous cystadeno CA

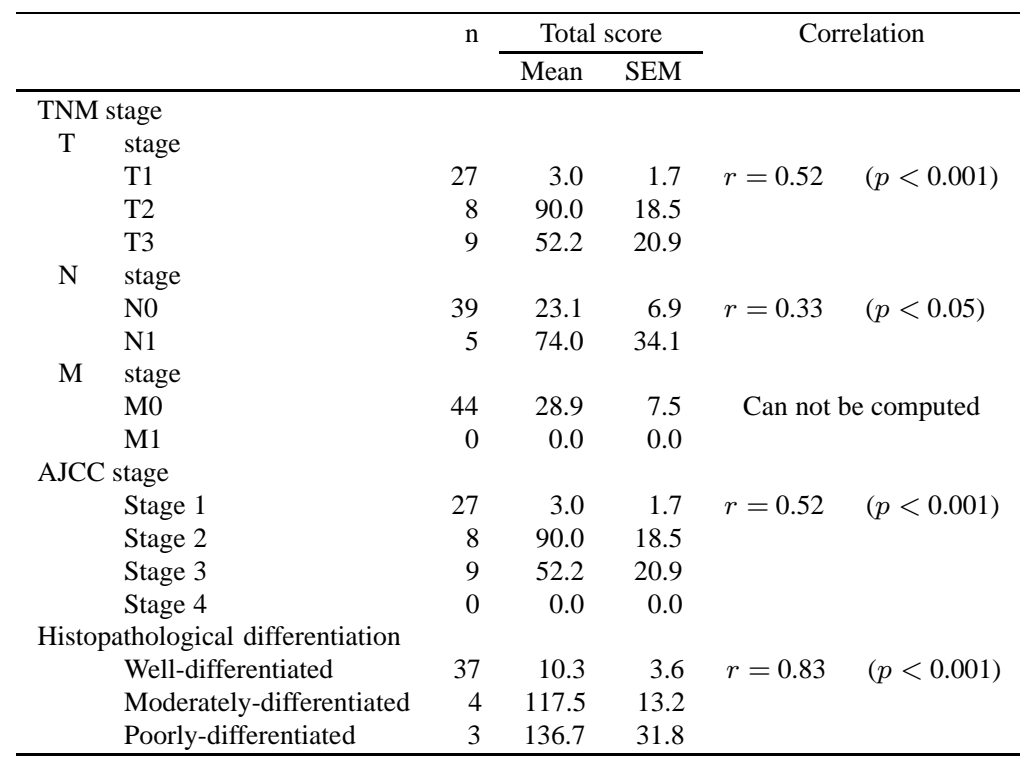

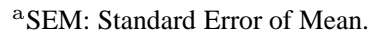
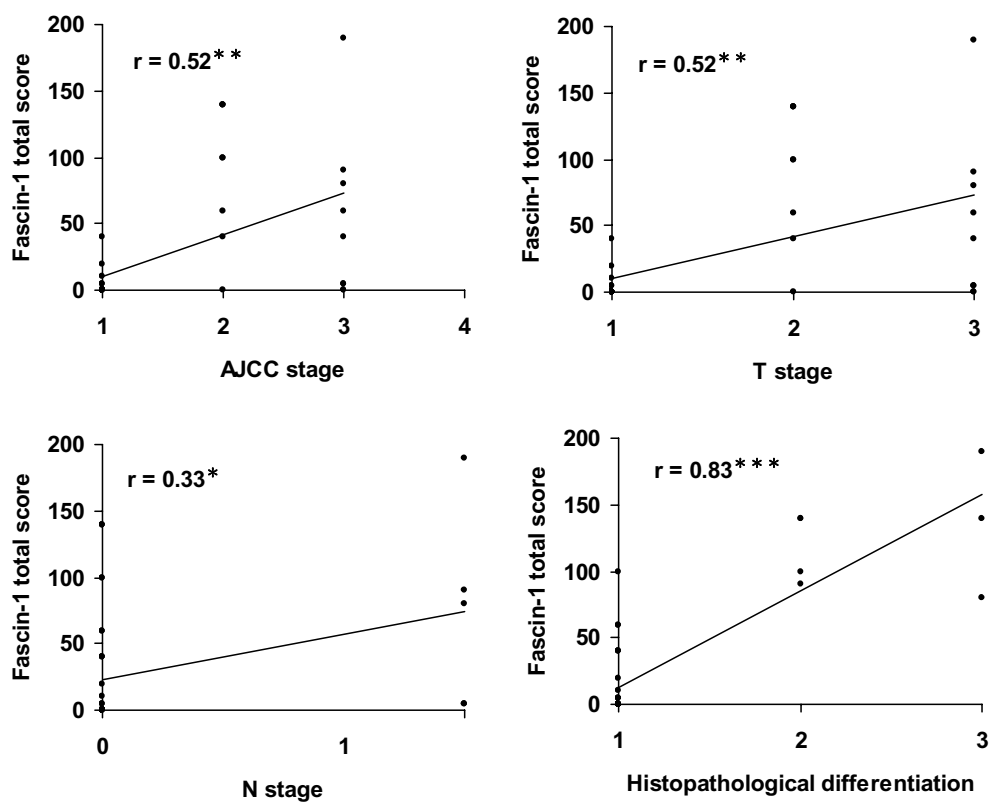

Fig. 2. Correlation of immunostaining scores of fascin-1 and clinicopathological parameters in mucinous cystadenocarcinoma. ${ }^{*}$ Indicates statistical significance of Pearson's correlation test $(p<0.05)$; $^{* *}$ indicates $p<0.01$; and ${ }^{* * *}$ indicates $\mathrm{p}$ value less than 0.001 .

M stages ( $p<0.01$; Table 3; Fig. 3). In addition, no significant relationships were seen between cortactin immunostaining scores and clinicopathological parameters in the other three ovarian epithelial carcinomas, which included mucinous, endometrioid and clear cell carcinoma (data not shown).

\subsection{Correlation of EGFR immunoscores in four ovarian carcinomas}

In our study, no significant relationships were seen between EGFR immunostaining scores and clinicopathological parameters in the four most common 
Table 3

Total score of cortactin immunostaining and clinicopathological parameters of serous cystadeno CA

\begin{tabular}{|c|c|c|c|c|c|c|}
\hline & & \multirow[t]{2}{*}{$n$} & \multicolumn{2}{|c|}{ Total score } & \multicolumn{2}{|c|}{ Correlation } \\
\hline & & & Mean & SEM & & \\
\hline \multicolumn{7}{|c|}{ TNM stage } \\
\hline \multirow[t]{4}{*}{$\mathrm{T}$} & stage & & & & & \\
\hline & $\mathrm{T} 1$ & 26 & 236.4 & 15.2 & $r=0.32$ & $(p<0.01)$ \\
\hline & $\mathrm{T} 2$ & 24 & 269.6 & 9.4 & & \\
\hline & T3 & 19 & 284.2 & 8.6 & & \\
\hline \multirow[t]{3}{*}{$\mathrm{N}$} & stage & & & & & \\
\hline & No & 50 & 262.6 & 9.2 & $r=-0.04$ & $(p=0.74)$ \\
\hline & N1 & 19 & 257.1 & 11.1 & & \\
\hline \multicolumn{7}{|c|}{ stage } \\
\hline & Mo & 67 & 260.8 & 7.5 & $r=0.03$ & $(p=0.84)$ \\
\hline & M1 & 2 & 270.0 & 30.0 & & \\
\hline \multicolumn{7}{|c|}{ AJCC stage } \\
\hline & Stage 1 & 21 & 235.8 & 18.1 & $r=0.18$ & $(p=0.14)$ \\
\hline & Stage 2 & 19 & 283.2 & 9.2 & & \\
\hline & Stage 3 & 27 & 264.6 & 9.2 & & \\
\hline & Stage 4 & 2 & 270.0 & 30.0 & & \\
\hline \multicolumn{7}{|c|}{ Histopathological differentiation } \\
\hline & Well-differentiated & 41 & 241.9 & 10.7 & $r=0.40$ & $(p<0.01)$ \\
\hline & Moderately-differentiated & 14 & 278.6 & 11.4 & & \\
\hline & Poorly-differentiated & 14 & 300.0 & 0.0 & & \\
\hline
\end{tabular}

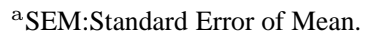

ovarian carcinomas, including serous, mucinous, endometrioid and clear cell carcinomas (data not shown). Our results suggest that EGFR may not be a good biomarker in evaluating the aggressiveness of ovarian epithelial carcinomas.

\subsection{Relationship of fascin-1 and cortactin expression with survival time}

We divided 69 serous cystadenocarcinoma and 44 mucinous cystadenocarcinoma cases that had received 5-year follow up into two groups based on cortactin and fascin-1 immunoscores, respectively. For cortactin immunoscores, those patients with higher expression ( $n=34$, immunostaining scores $\geqslant 250$ ) were in group one, the remaining cases had lower immunoreactivity ( $n=35$, immunostaining score $<250$ ) were in group two. The absolute number of patients that succumbed to disease in five years and the median survival time for higher expression of cortactin are 16 cases and 45.3 months, and for lower expression of cortactin are 11 cases and 47.4 months.

The two groups were also divided incuding higher fascin-1 expression ( $n=11$, immunostaining scores $>0$ ) and negative fascin- 1 immunoactivity ( $n=33$, immunostaining scores $=0$ ). The absolute number of patients that succumbed to disease in five years and the median survival time for higher expression of fascin are
7 cases and 31.3 months, and for negative expression of fascin are 9 cases and 54.4 months.

Using cortactin and fascin-1 immunoscores as variable parameters, higher scores in fascin-1 was significantly associated with higher mortality ( $\mathrm{p}$ value $<$ 0.001 ; Fig. 4). However, there was no significant difference in survival rate between higher or lower expression of cortactin (Fig. 5).

\section{Discussion}

At present, the emergence of cancer is a complex multi-step process in which the activation of oncogenes and inactivation of tumor suppressor genes act synergistically to produce the malignant phenotype. Tumor metastasis is also a complex process that involves direct invasion of tumor cells, infiltration into lymphovascular channels, survival in circulation, extravasation, and growth at secondary sites [3]. Although these mechanisms are poorly understood, we do know that one requirement is enhancement of cell motility. In fact, enhanced movement of cancer cells has been reported to correlate with greater metastatic potential in animal models and poorer prognosis in human cancers [24].

Fascin-1, a 55-kDa globular protein, aggregates Factin into parallel bundles to rearrange the cytoskeleton and promote cellular motility [21]. Fascin-1 plays important roles in cellular protrusion and migration, and 

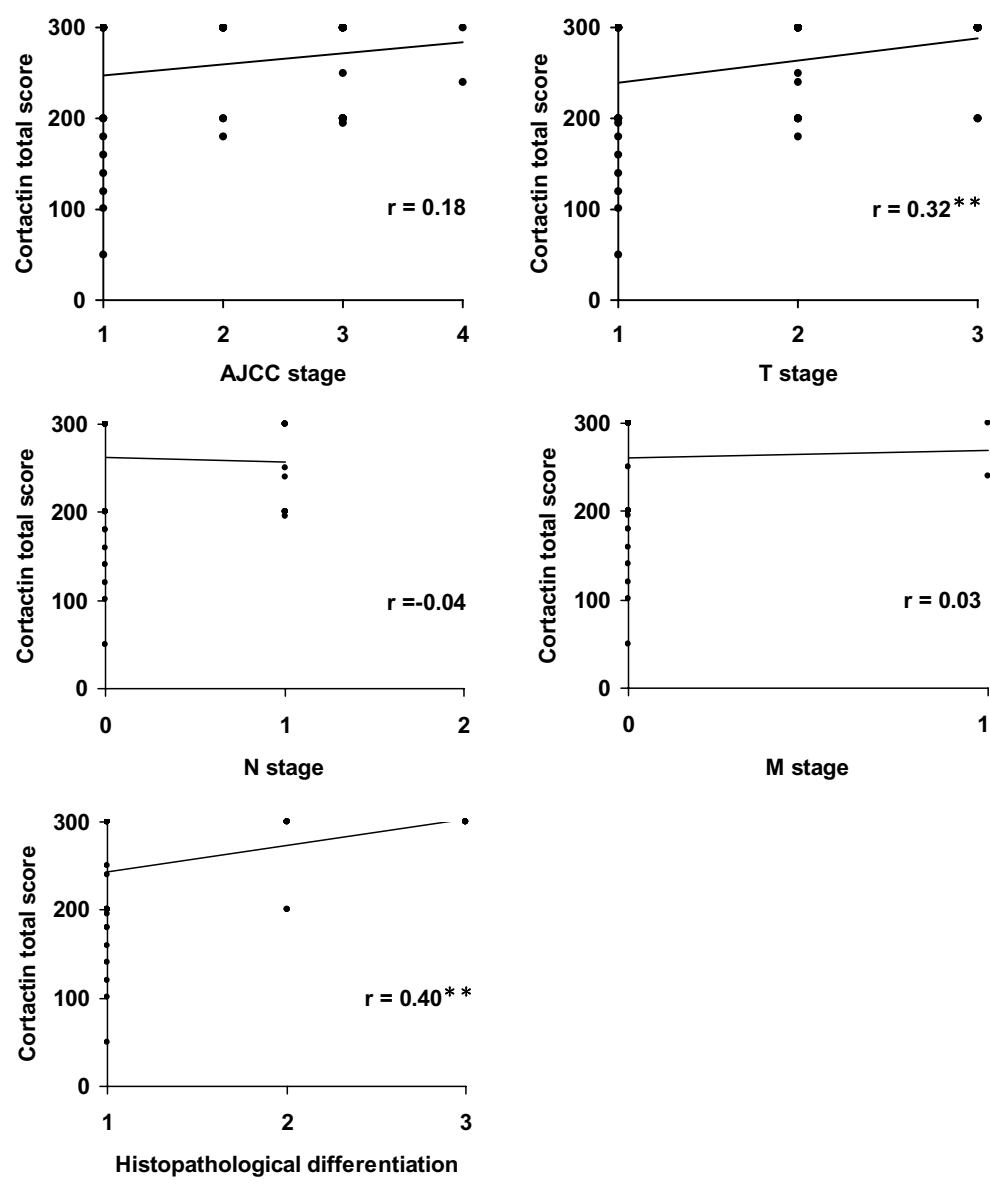

Fig. 3. Correlation of immunostaining scores of cortactin and clinicopathological parameters in serous cystadenocarcinoma. ${ }^{* *}$ Indicates statistical significance of Pearson's correlation test $(p<0.01)$.

extracellular matrix adhesion [2]. The gene encoding fascin-1 in humans is located at chromosome 7q22 [14]. Fascin-1 is either expressed at very low levels or is absent in normal ovarian epithelia. Over-expression of fascin-1 is associated with tumor progression and invasion in skin, lung, breast, ovarian, colon, urinary bladder, brain and gastric malignancies $[11,13,17,19$, 25,26,28,33,34].

Our results suggest that expression of fascin-1 may be effective in predicting tumor clinicopathological parameters of ovarian mucinous cystadenocarcinoma in Chinese women. Average immunostaining scores for fascin-1 have a significant positive correlation with $\mathrm{T}$, $\mathrm{N}$, and AJCC stages, but not with $\mathrm{M}$ stage of mucinous cystadenocarcinoma. However, in our study, no M1-stage case was included, which makes it difficult to show statistical significance. In addition, higher fascin1 immunostaining scores are significantly associated with poorer survival rate as demonstrated in our study.
Cortactin regulates the actin cytoskeleton through its involvement in several processes, including cell motility, adhesion, polarization, contraction, and others $[9$, $35,40]$. The activation of actin-related (Arp) 2/3 protein complex and neuronal Wiscott-Aldrich syndrome protein (N-Wasp) by cortactin nucleates actin polymerization and promotes cellular motility. Cortactin is a p80/p85 multidomain actin filament-binding protein [32]; it was first identified as an src kinase substrate in chicken fibroblasts [41]. Human cortactin maps to chromosome 11q13 [22]. Amplification of chromosome $11 \mathrm{q} 13$ has been reported in several human carcinomas as has increased expression of cortactin [37]. Over-expression of cortactin induces cell motility and migration, inhibits cell-cell adhesion, and accelerates tumor spreading [36]. In addition, the effects of cortactin may be related to expression of E-cadherin and its effects on intercellular adhesion [15,20,38]. In some in vitro studies, cortactin over-expression induced tu- 


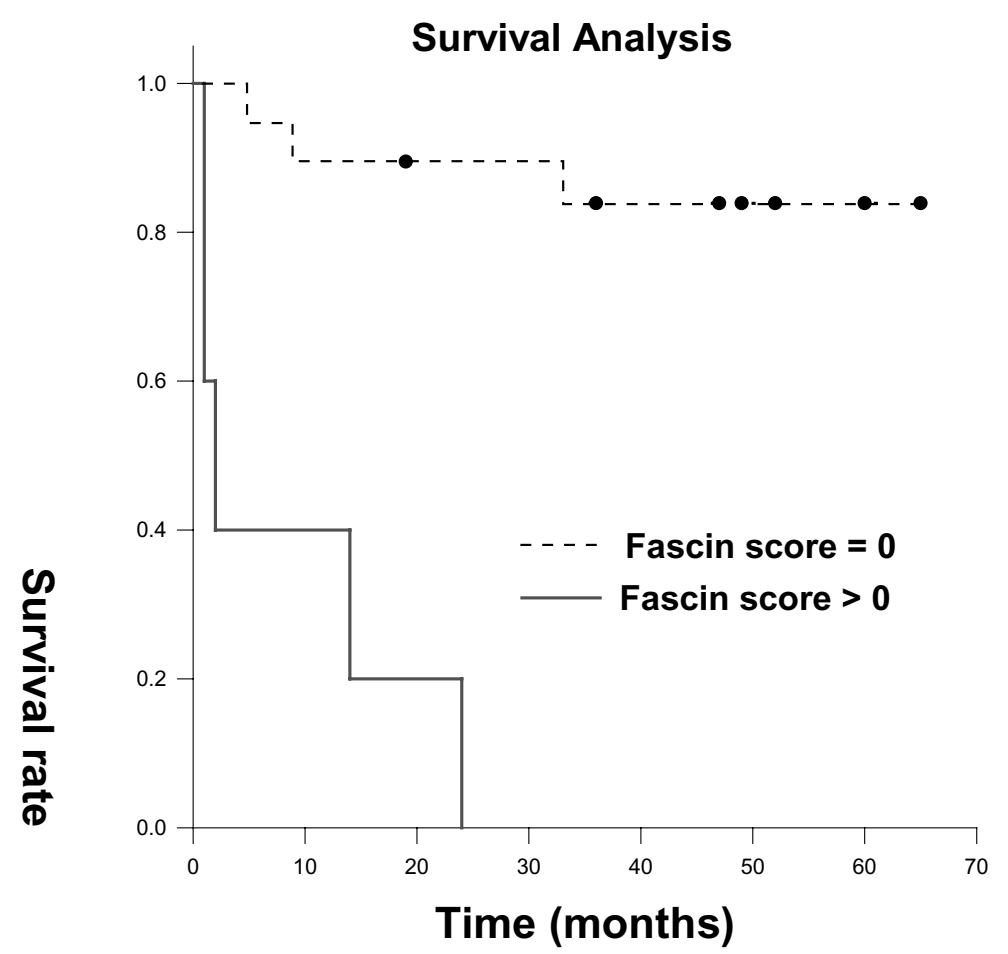

Fig. 4. Overall survival of 44 patients with mucinous cystadenocarcinoma. Higher fascin-1 immunostaining scores were associated with poorer survival. Survival rates were analyzed using the Kaplan-Meier survival test $(p<0.001)$.

mor invasion, and metastasis has been shown to be associated with esophageal and head/neck squamous cell carcinomas [22,29]. However, direct evidence is still lacking to establish a relationship between cortactin over-expression and tumor progression and metastasis in ovarian carcinomas. Our current results demonstrate that cortactin is over-expressed in the four most common ovarian carcinomas in Chinese women, and higher immunostaining scores of cortactin are associated with more advanced $\mathrm{T}$ stage and histological differentiation in serous cystadenocarcinoma (Table 3, Fig. 3).

EGFR is one of a family of receptors that help regulate cell growth, division, and death. Normal epithelial cells contain two copies of the EGFR gene and produce low levels of EGFR protein on the cell surface. In a variety of cancers, increased amounts of EGFR protein are present in tumor tissue. This can be due to amplification (too many copies of the gene are produced), over-expression (an increased amount of the protein is produced), and/or decreased protein destruction. Tumors with increased EGFR protein tend to grow more aggressively, are more likely to metastasize, and are more resistant to standard chemotherapies. Patients with these tumors tend to have poorer outcomes.
In our study, EGFR is over-expressed in four ovarian carcinomas in Chinese women, but over-expression of EGFR was not associated with aggressive clinicopathological parameters in these four ovarian carcinomas. To our knowledge, this is the first report to evaluate the association between cortactin, fascin-1 and EGFR expression and tumor progression in the four most common ovarian epithelial carcinomas.

In conclusion, higher fascin-1 immunostaining scores are associated with poorer tumor differentiation, more-advanced TNM stages and shorter survival time in mucinous cystadenocarcinoma. Similarly, we found a correlation between higher immunostaining scores of cortactin and $\mathrm{T}$ stages and histological differentiation in serous cystadenocarcinoma. Accordingly, our results may support the hypothesis that fascin-1 and cortactin are important factors in migration or invasion of some ovarian epithelial carcinomas. Although unknown mechanisms still exist in tumor progression, we demonstrated that fascin-1 is a satisfactory biomarker for predicting clinical outcome in mucinous cystadenocarcinoma. Therefore, the development of pharmacological agents to target the fascin-1 pathway may prolong survival time and slow tumor progression in patients with mucinous cystadenocarcinoma. 


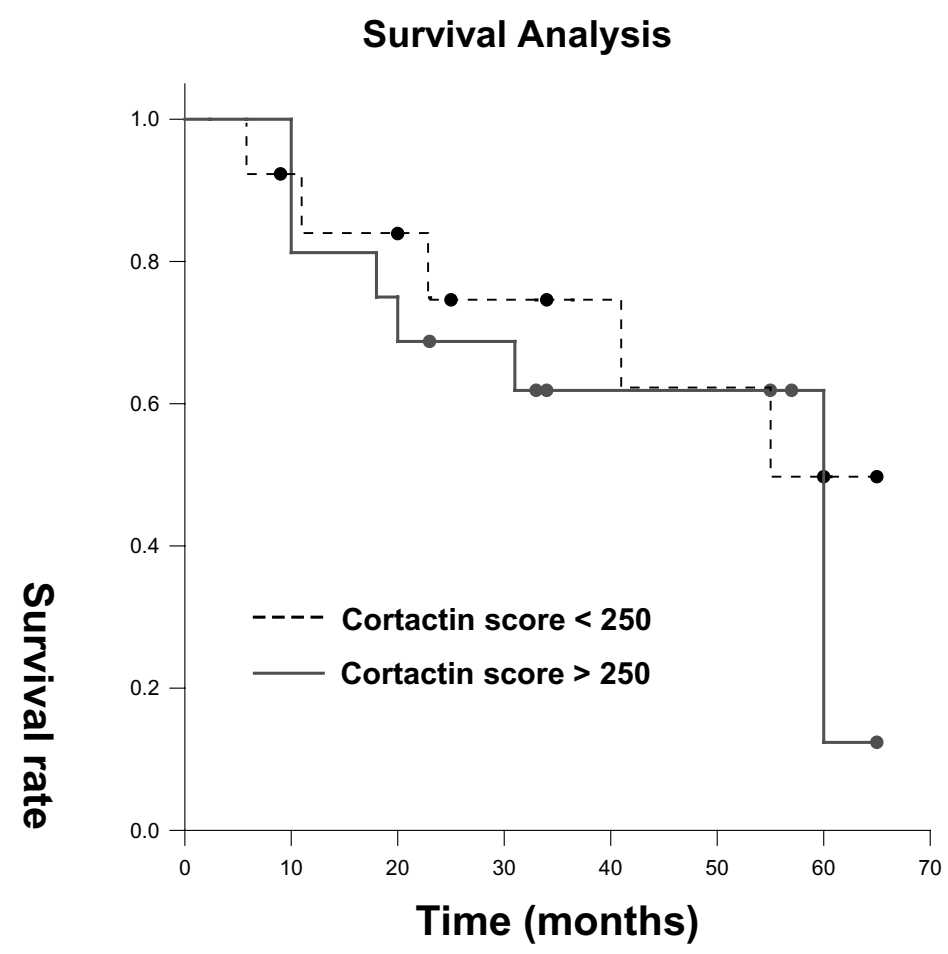

Fig. 5. Overall survival of 69 patients with serous cystadenocarcinoma. Higher cortactin immunostaining scores were not associated with poorer survival rate. Survival rates were analyzed using the Kaplan-Meier survival test $(p>0.05)$.

\section{Acknowledgments}

This study was supported by a grant from Tri-Service General Hospital, TSGH-C97-4-S05, Taiwan.

\section{References}

[1] J.C. Adams, Roles of fascin in cell adhesion and motility, Curr Opin Cell Biol 16 (2004), 590-596.

[2] J.C. Adams, Fascin protrusions in cell interactions, Trends Cardiovasc Med 14 (2004), 221-226.

[3] S. Anzavoorian, A.N. Murphy, W.G. Steler-Stevenson and L.A. Liotta, Molecular aspects of tumor cell invasion and metastasis, Cancer 71 (1993), 1368-1383.

[4] T. Bauknecht, I. Janz, M. Kohler and A. Pfleiderer, Human ovarian carcinomas: correlation of malignancy and survival with the expression of epidermal growth factor receptors and EGFR-like factors, Med Oncol Tumor Pharmacother 6 (1989), 121-127.

[5] T. Bauknecht, Birmelin and F. Kommoss, Clinical significance of oncogenes and growth factors in ovarian carcinomas, $J$ Steroid Biochem Mol Biol 37 (1990), 855-862.

[6] A. Berchuck, G.C. Rodriguez, A. Kamek, R.K. Dodge, J.T. Soper, D.L. Clarke-Pearson et al., Epidermal growth factor receptor expression in normal ovarian epithelium and ovarian cancer, Am J Obstet Gynecol 164 (1991), 669-674.

[7] E. Brown, M. Stewart, T. Rye, A. Al-Nafussi, A.R. Williams, M. Bradburn et al., Carcinosarcoma of the ovary: 19 years of prospective data from a single center, Cancer 100 (2004), 2148-2153.

[8] G. Carpenter, Epidermal growth factor, Annu Rev Biochem 48 (1979), 193-216.

[9] R.J. Daly, Cortactin signaling and dynamic actin networks, Biochem J 382 (2004), 13-25.

[10] B. Davidson, I. Goldberg, A. Berner, G.B. Kristensen and R. Reich, EMMPRIN (extracellular matrix metalloproteinase inducer) is a novel marker of poor outcome in serous ovarian carcinoma, Clin Exp Metastasis 20 (2003), 161-169.

[11] V.N. Goncharuk, J.S. Ross and J.A. Carlson, Actin-binding protein fascin expression in skin neoplasia, J Cutan Pathol 29 (2002), 430-438.

[12] M.E. Greenberg and E.B. Ziff, Stimulation of 3T3 cells induces transcription of the c-fos proto-oncogene, Nature (London) 311 (1984), 433-438.

[13] A. Grothey, R. Hashizume, A.A. Sahin and P.D. McCrea, Fascin, an actin-bundling protein associated with cell motility, is upregulated in hormone receptor negative breast cancer, $\mathrm{Br}$ J Cancer 83 (2000), 870-873.

[14] Y. Hashimoto, M. Skacel and J.C. Adams, Roles of fascin in human carcinoma motility and signaling: prospects for a novel biomarker? Int J Biochem Cell Biol 37 (2005), 1787-1804.

[15] F.M. Helwani, E.M. Kovacs, A.D. Paterson, S. Verma, R.G. Ali, A.S. Fanning, S.A. Weed et al., Cortactin is necessary for E-cadherin-mediated contact formation and actin reorganization, J Cell Biol 164 (2004), 899-910.

[16] S.C. Henzen-Longmans, E.M. Beins, J.G. Klyn, M.E. van den Burg and J.A. Foekens, Epidermal growth factor receptor: correlation of immunohistochemistry with ligand binding assay, Br J Cancer 66 (1992), 1015-1021. 
[17] W. Hu, P.D. McCrea, M. Deavers, J.J. Kavanagh, A.P. Kudelka and C.F. Verschraegen, Increased expression of fascin, motility associated protein, in cell cultures derived from ovarian cancer and in borderline and carcinomatous ovarian tumors, Clin Exp Metastasis 18 (2000), 83-88.

[18] H. Itamochi, J. Kigawa, T. Sugiyama, Y. Kikuchi, M. Suzuki and N. Terakawa, Low proliferation activity may be associated with chemoresistance in clear cell carcinoma of the ovary, Obst \& Gynecol 100 (2002), 281-287.

[19] A.U. Jawhari, A.Buda, M. Jenkins, K. Shehzad, C. Sarraf, M. Noda et al., Fascin, an actin-bundling protein, modulates colonic epithelial cell invasiveness and differentiation in vitro, Am J Pathol 162 (2003), 69-80.

[20] E.M. Kovacs, M. Goodwin, R.G. Ali, A.D. Paterson and A.S. Yap, Cadherin-directed actin assembly: E-cadherin physically associates with the Arp2/3 complex to direct actin assembly in nascent adhesive contacts, Curr Biol 12 (2002), 379-382.

[21] N. Kureishy, V. Sapountzi, S. Prag, N. Anilkumar and J.C. Adams, Fascins and their roles in cell structure and function, Bio Essays 24 (2002), 350-361.

[22] M.L. Luo, X.M. Shen, Y. Zhang, F. Wei, X. Xu, Y. Cai, $X$. Zhang et al., Amplification and overexpression of CTTN (EMS1) contribute to the metastasis of esophageal squamous cell carcinoma by promoting cell migration and anoikis resistance, Cancer Res 66 (2006), 11690-11699.

[23] C.J. Ormandy, E.A. Musgrove, R. Hui, R.J. Daly and R.L. Sutherland, Cyclin D1, EMS1 and 11q13 amplification in breast cancer, Breast Cancer Res Treat 78 (2003), 323-335.

[24] A.W. Partin, J.S. Shoeniger, L.L. Mohler and D.S. Coffey, Further analysis of cell motility: correlation of motility with metastatic potentials, Proc Natl Acad Sci 86 (1989), 12541258.

[25] G. Pelosi, F. Pasini, F. Fraggetta, U. Pastorino, A. Iannucci, P. Maisonneuve et al., Independent value of fascin immunoreactivity for predicting lymph node metastases in typical and atypical pulmonary carcinoids, Lung Cancer 42 (2003), 203213.

[26] G. Pelosi, U. Pastorino, F. Pasini, P. Maissoneuve, F. Fraggetta, A. Iannucci et al., Independent prognostic value of fascin immunoreactivity in Stage I non-small cell lung cancer, $\mathrm{Br} J$ Cancer 88 (2003), 537-547.

[27] J.M. Piek, P. Kenemans and R.H. Verheijen, Intraperitoneal serous adenocarcinoma: a critical appraisal of three hypotheses on its cause, Am J Obst \& Gynecol 191 (2004), 718-732.

[28] A.A. Roma and R.A. Prayson, Fascin expression in 90 patients with glioblastoma multiforme, Ann Diagn Pathol 9 (2005), 307-311.

[29] B.L. Rothschild, A.H. Shim, A.G. Ammer, L.C. Kelley, K.B. Irby et al., Cortactin overexpression regulates actin-related protein $2 / 3$ complex activity, motility, and invasion in carcinomas with chromosome 11q13 amplification, Cancer Res 66 (2006), 8017-8025.

[30] E. Rozengurt, Stimulation of $\mathrm{Na}^{+} / \mathrm{K}^{+}$pump activity and DNA synthesis in quiescent cultured cells, Adv Enzyme Regul 19 (1981), 61-85.

[31] E. Schuuring, The involvement of the chromosome 11q13 region in human malignancies: cyclin D1 and EMS1 are two new candidate oncogenes - a review, Gene 159 (1995), 83-96.

[32] E. Schuuring, E. Verhoeven, S. Litvinov and R.J. Michslides, The product of the EMS1 gene, amplified and overexpressed in human carcinomas, is homologous to a v-src substrate and is located in cell-substratum, contact sites, Mol Cell Biol 13 (1993), 2891-2898.

[33] W.C. Tsai, J.S. Jin, W.K. Chang, D.C. Chan, M.K. Yeh, Y.C. Chao et al., Association of cortactin and fascin-1 expression in gastric adenocarcinoma: correlation with clinocopathological parameters, Disease Markers 55 (2007), 955-962.

[34] G.X. Tong, H.Yee, L. Chiriboga, O. Hernandes and J. Waisman, Fascin-1 expression in papillary and invasive urothelial carcinomas of the urinary bladder, Human Pathology 36 (2005), 741-746.

[35] A.G. van Rossum, J. Gibcus, J. van der Wal and E. Schuuring, Cortactin overexpression results in sustained epidermal growth factor receptor signaling by preventing ligand-induced receptor degradation in human carcinoma cells, Brease Cancer Res 7 (2005), 235-237.

[36] A.G. van Rossum, W.H. Moolenaar and E. Schuuring, Cortactin affects cell migration by regulating intercellular adhesion and cell spreading, Exp Cell Res 312 (2006), 1658-1670.

[37] A.G. van Rossum, E. Schuuring-Scholtes, V. van Buuren-van Seggelen, P.M. Kluin and E. Schuuring, Comparative genome analysis of cortactin and HS1: the significance of the F-actin binding repeat domain, BMC Genomics 6 (2005), 15.

[38] S. Verma, A.M. Shewan, J.A. Scott, F.M. Helwani, N.R. den Elzen et al., Arp2/3 activity is necessary for efficient formation of E-cadherin adhesive contacts, J Biol Chem 279 (2004), 34062-34070.

[39] A.M. Weaver, A.V. Karginov, A.W. Kinley, S.A. Weed, Y. Li, J.T. Parsons and J.A. Cooper, Cortactin promotes and stabilizes Arp2/3-induced actin filament network formation, Curr Biol 11 (2001), 370-374.

[40] S.A. Weed and J.T. Parsons, Cortactin: coupling membrane dynamics to cortical actin assembly, Oncogene 20 (2001), 6418-6434.

[41] H. Wu, A.B. Reynolds, S.B. Kanner, R.R. Vines and J.T. Parsons, Identification and characterization of a novel cytoskeleton-associated pp60src substrate, Mol Cell Biol 11 (1991), 5113-5124.

[42] J.J. Xie, L.Y. XU, H.H. Zhang, W.J. Cai and R.Q. Mai, Role of fascin in the proliferation and invasiveness of esophageal carcinoma cells, Biochem Biophys Res Commum 337 (2005), 355-362.

[43] S. Yamashiro, Y. Yamakita, S. Ono and F. Matsumura, Fascin, an actin-bundling protein, induces membrane protrusions and increases cell motility of epithelial cells, Mol Biol Cell 9 (1998), 993-1006 


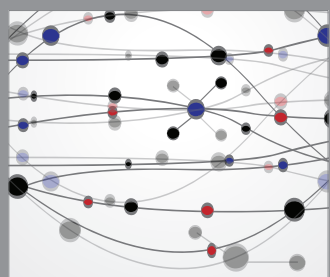

The Scientific World Journal
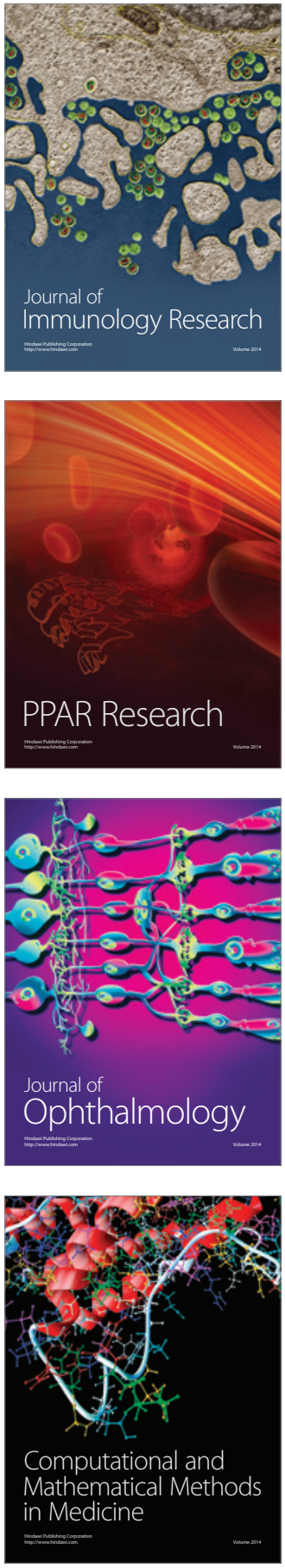

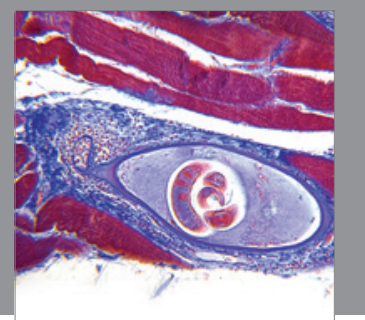

Gastroenterology

Research and Practice
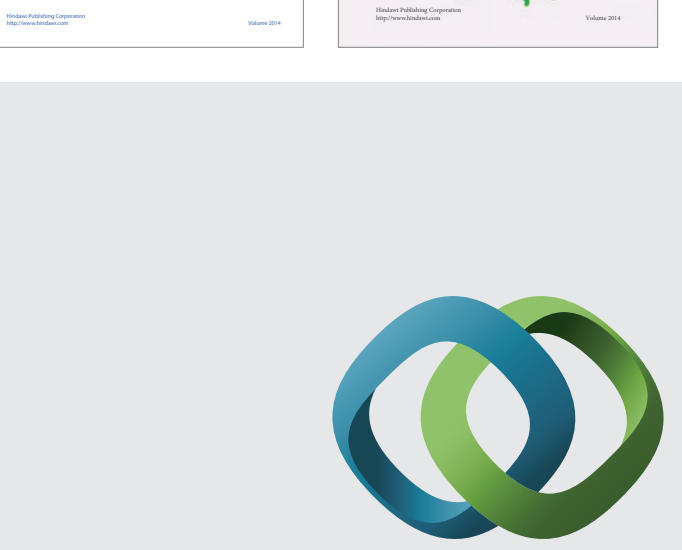

\section{Hindawi}

Submit your manuscripts at

http://www.hindawi.com
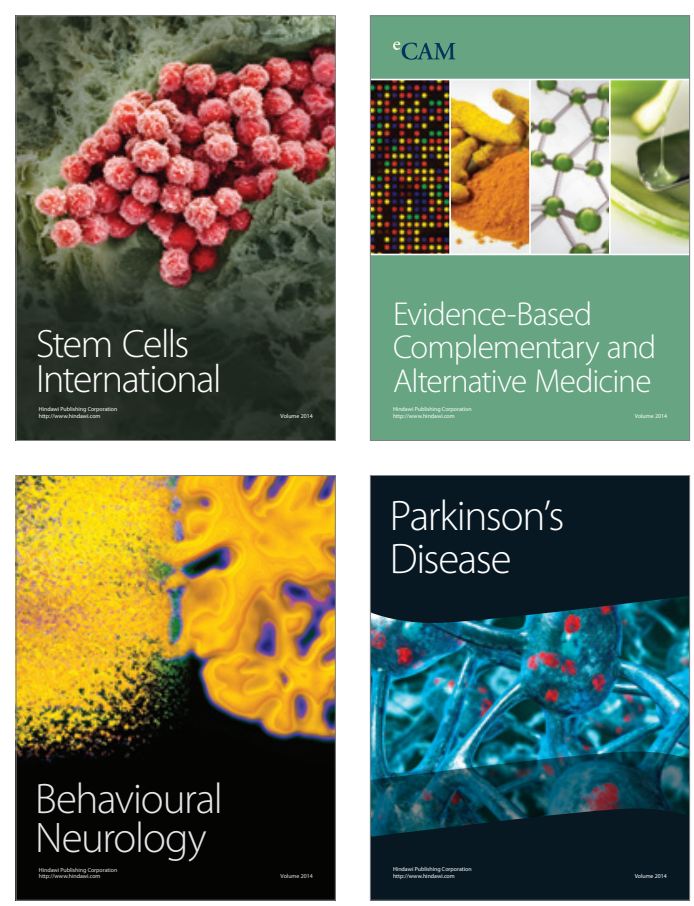

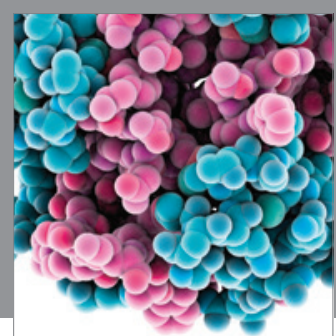

Journal of
Diabetes Research

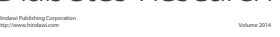

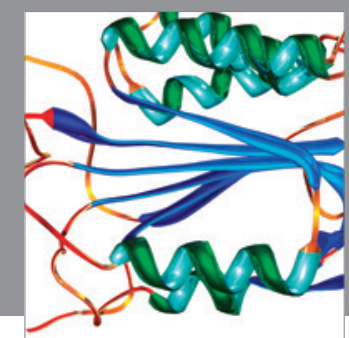

Disease Markers
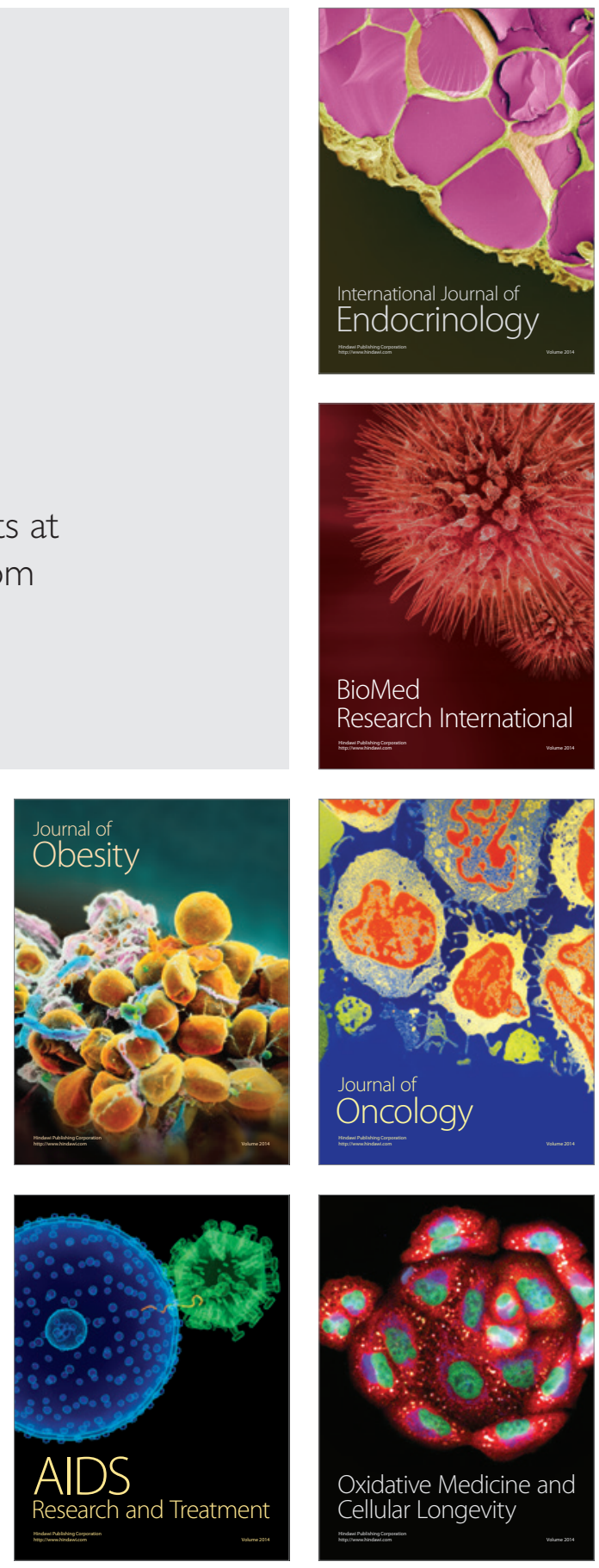